\title{
Storage and the Shift to Low Carbon Energy
}

\author{
Taco Niet, Lawrence Pitt, Andrew Rowe, and Peter Wild
}

\begin{abstract}
This work looks at the impact of assumptions made regarding efficiency of storage systems used with variable energy resources and how this applies to a solar PV installation. To find the optimal storage system to work with the cyclic solar output, a linear optimization model is implemented using OSeMOSYS.

With $100 \%$ efficient, free storage, with no capacity restrictions, it is possible to get down to almost $5 \mathrm{GW}$ of required solar installed capacity, but it requires 1.1 TWh of $100 \%$ efficient storage. Existing pumped hydro storage facilities have efficiencies between 70 and $80 \%$, which increase these numbers to $7 \mathrm{GW}$ and $1.2 \mathrm{TWh}$.

With a storage model based on the worlds largest pumped hydro facility between 20 and $25 \mathrm{GW}$ of installed solar capacity are required plus between 15 and $30 \mathrm{GWh}$ of storage capacity to meet the $1 \mathrm{GW}$ load. The capital infrastructure required to allow a solar installation to meet that of a baseload plant is therefore around an order of magnitude larger than what is commonly assumed. A shift away from fossil fuels to renewable/variable energy resources will require more infrastructure than indicated by simply considering the capacity factor of the energy source.
\end{abstract}

Index Terms-Solar energy, storage, renewable energy, efficiency, climate change.

\section{INTRODUCTION}

Solar panels, wind turbines, hydroelectric facilities and other such renewable energy resources are often touted as being a viable source of low-emission energy/electricity since the operating emissions are low for these technologies. The build emissions related to these technologies impact their ability to provide low life-cycle emissions. One study by Myhrvold and Caldeira (2012) discusses the fact that the emissions from the building of solar, wind and hydroelectric power plants are a significant portion of the lifecycle emissions, and they discuss how this affects the ability of these technologies to reduce emissions when compared with coal fired power generation [1]. They analyse the build-out of solar, wind, nuclear, natural gas, hydro and coal with carbon capture and storage (CCS) technologies to replace the approximately $1 \mathrm{TW}$ of existing coal power plants around the world by including the impacts of build emissions. They conclude that only solar, hydro and nuclear can have a significant impact in reducing emissions, and therefore mitigate climate change cause by electricity generation

Manuscript received September 30, 2014; revised January 18, 2015. This work was supported in part by funding from the Pacific Institute for Climate Solutions (PICS). Thanks to Jonn Axsen of the Energy and Materials Research Group at SFU for his review of the initial draft of the paper.

Taco Niet is with the British Columbia Institute of Technology (BCIT), Burnaby, BC, Canada (e-mail: tniet@bcit.ca).

Lawrence Pitt, Andrew Rowe, and Peter Wild are with the Institute for Integrated Energy Systems (IESVic) at the University of Victoria, Victoria, BC, Canada. worldwide.

Their build out plans for nuclear, natural gas, hydro and coal with CCS are potentially feasible, as these technologies can be built up to a 1TW of installed capacity, and would then perform in a similar manner as the plant they would be replacing. There are still significant issues with their build out plan, not the least of which is that building $1 \mathrm{TW}$ of power generation in 40 years is quite an ambitious goal, as discussed by Socolow [2]. In addition, for hydroelectricity, it is not clear if there are enough locations worldwide to enable $1 \mathrm{TW}$ of capacity to be built.

For solar thermal, solar PV and wind, Myhrvold and Caldeira state correctly that the output is weather dependent. They go on to say that they "compare plants scaled to a power plant with $1 \mathrm{GWe}$ net output averaged over a year, which is equivalent to $8.76 \mathrm{TWh}$ of total electrical energy per year" [1, p. 18 Supplement]. In making this statement they ignore the time of production, which is akin to saying $100 \%$ efficient, infinite storage is available. This is a common assumption in the public media as well as in some scholarly articles as illustrated by a similar assumption in a recent article, where the author assumes that the solar installation currently in California can be scaled to provide all the electrical needs of the state while ignoring the time of production [3]. Many other examples can be found.

The current work looks at the assumption of infinite, $100 \%$ efficient storage. Specifically, it looks at the storage that would allow a solar installation to replace the power from a 1 GW coal plant. Three research objectives are considered:

1) How much $100 \%$ efficient storage is required to allow a solar installation that produces 8760 TWh of electricity annually to provide a steady $1 \mathrm{GW}$ of output (similar to a coal fired power plant)?

2) How do storage efficiency values impact the installation requirements?

3) How does limited storage capacity affect the required installation of solar?

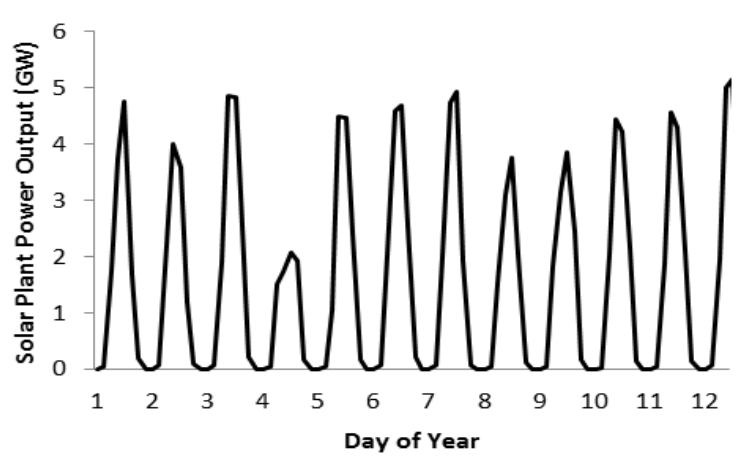

Fig. 1. Cyclic daily power output from solar [4].

Fig. 1 shows the daily cycle of power output from the simulated solar panel installation and illustrates the need for storage. As can be seen from this figure, daily storage is 
required to allow the lights to stay on at night when the solar panel is not producing any power. Storage is also required to deal with weather related variations in output and seasonal variations (summer/winter).

\section{OSEMOSYS OPEN SOURCE ENERGY MODELLING SYSTEM}

To find the optimal storage system to work with the cyclic solar output, a linear optimization model is implemented using the OSeMOSYS Open Source Energy Modelling System [5], [6]. OSeMOSYS is an energy system optimization tool that functions in a similar way to other linear optimization tools, but is open source and transparent, uses the freely available GNU MathProg solver [7], and is intended to be simple to learn. The main features of OSeMOSYS that make it an appropriate modelling tool for this project is that it is a linear optimization model (since we are trying to determine the minimum storage and system requirements), it allows for the modelling of storage and variable resources, and it allows for explicit technology representation.

The conceptual structure of OSeMOSYS with all its functional blocks is shown in Fig. 2. This figure shows the different constraints categories implemented in OSeMOSYS. As can be seen from this figure, OSeMOSYS is a very general energy system modelling tool that has a broad range of capabilities.

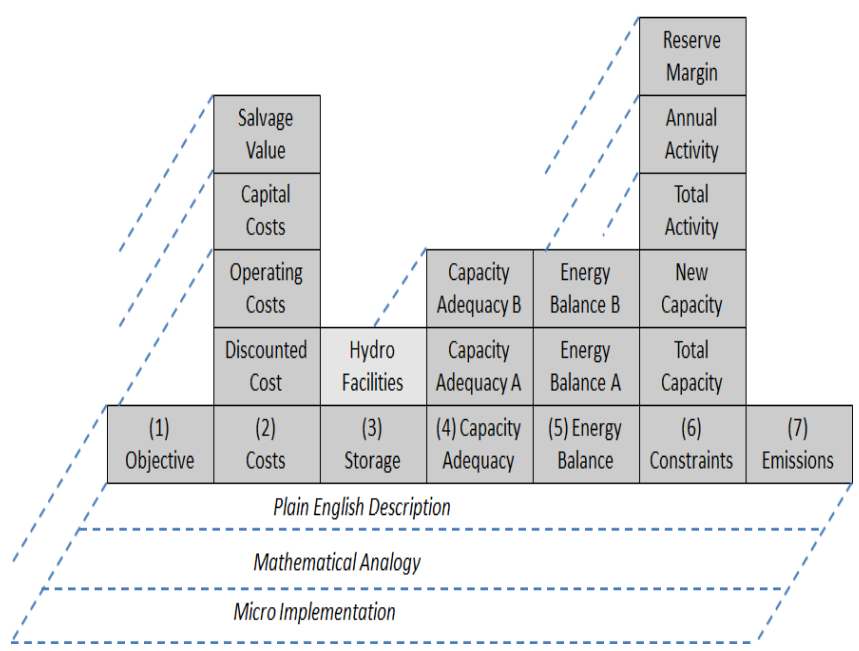

Fig. 2. OSeMOSYS conceptual structure (after [5], [6]).

Only a sub-set of the capabilities of OSeMOSYS were used for this study. Specifically, the variable resource, the storage model, and the demand model were used while other aspects such as the emissions tracking, detailed aspects of cost, reserve margin, discount rates were not used as they were not necessary to answer the research questions.

A modification to OSeMOSYS was implemented to enable the system to answer research question number 3 , the effect of limited storage. The storage implementation in the base OSeMOSYS model allows for the restriction of the input and output power, but not of the storage capacity. To allow the model to restrict the size of the available storage capacity a parameter named "StorageMaxCapacity" was created, and a constraint was implemented that requires the variable "StorageUpperLimit" to be less than or equal to
"StorageMaxCapacity". This allows the modeller to specify a maximum level of stored energy in the model as in (1).

\section{StorageMaxCapacity $\geq$ StorageUpperLimit}

\section{Modelling Methodology AND Structure}

TABLE shows the assumptions Myhrvold and Caldeira made to get to $5 \mathrm{GW}$ of installed solar capacity to replace a 1 GW coal plant. As noted in the introduction, the current work looks only at the validity of assumption \#2, although the other assumptions are significant and seriously impact resource/generation technology feasibility.

TABLE I: AssumPtiONS IMPLIED BY METHODOLOGY IN [1]

\begin{tabular}{cl}
\hline \hline \multicolumn{1}{c}{ Myhrvold and Caldeira Assumptions } \\
\hline 1. & $\begin{array}{l}\text { A } 5 \text { GWe (20\% capacity factor) Solar Facility can produce } \\
8760 \mathrm{GWh} \text { of electricity over the course of a year (similar to } \\
\text { a 1 GW coal plant). }\end{array}$ \\
2. & $\begin{array}{r}\text { It does not matter when the GWh are generated. } \\
\text { a. Infinite (Free) Storage Capacity }\end{array}$ \\
b. 100\% Storage Efficiency \\
3. There are locations available for installation. \\
4. Transmission is free and 100\% efficient.
\end{tabular}

To match the assumptions made by Myhrvold and Caldeira, we assume that there are infinite suitable locations available for installation, and that transmission is free and $100 \%$ efficient. Both of these assumptions are questionable, but are reasonable as a first cut at investigating the scale of the problem. If storage turns out not to be a major restriction, and if suitable scale storage systems are available, the model can be expanded to look at those assumptions in the future.

The basic model structure implemented in OSeMOSYS is shown in Fig. 3. To allow the model to reasonably track the daily, weekly and seasonal variations in solar output, the year was broken into 2920 time periods of three hours each. Each period represented a contiguous set of three hours. This provided a reasonable balance between the computational complexity of the model (and longer run times) and the representation of the cyclic nature of the solar resource. A constant $1 \mathrm{GW}$ demand is implemented to model the output of a coal fired power plant operating continuously over the year.

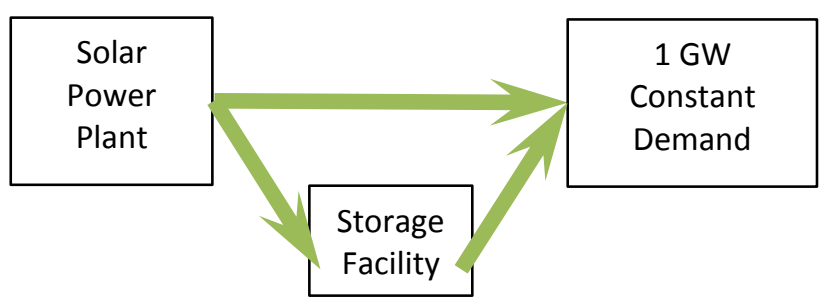

Fig. 3. Model structure implemented in OSeMOSYS.

The solar power plant is modelled based on insolation values from a typical meteorological year for Flagstaff, Arizona obtained from the National Solar Database [4]. The insolation data is converted to a percent of the yearly maximum value. This percent can be used in OSeMOSYS as a 'capacity factor' for the solar power plant, scaling the output for each time period based on the availability of the solar resource, from 0 when there is no sun to 1 when the insolation is at its maximum. Since Myhrvold and Caldeira 
assumed a $20 \%$ capacity factor (a 5 GWe plant can output 8.78 TWh of electricity), and the average output, based on the NREL data set from Flagstaff is $18 \%$ (producing only 7.88 TWh in the year), the data was scaled up slightly to match. This was considered a conservative approach. Actual capacity factors for existing solar power facilities in the United States range from $2.5 \%$ in the winter to over $30 \%$ in the summer, with an average of around $20 \%$ [8].

Storage is modelled as an input efficiency, a stored capacity, and output efficiency. When considering less than $100 \%$ efficient storage, the losses are divided evenly onto the input and output. For example, a 90\% round trip efficient storage assumption is modelled as a $94.9 \%$ input efficiency and a $94.9 \%$ output efficiency (each the square root of 0.9 ), such that $90 \%$ of the energy into the system is available at the output at a later time. This would not be the case for any real storage system but is a good first order approximation.

Other factors, such as losses in the system over time (such as self-discharge of batteries, or leakage over time in a pumped hydro facility) are not considered in the model at this time. These factors could be implemented if the initial results show that they may significantly impact the results.

To allow OSeMOSYS to determine the minimum storage level required, the cost of solar was set at a nominal value while the cost of storage was set to nearly free. For the overall model, the entire storage system ended up costing somewhere on the order of $0.01 \%$ of the overall system. The nominal cost of the solar installation ensured that the model results showed the lowest solar installation that can meet the demand while the nearly free cost of storage capacity ensured that the storage capacity results were minimized for each run.

For all runs, the initial storage level was set to 6 hours of storage to allow the model to get to the first day of solar insolation. As such, the starting value of storage was set to 6 GWh for runs with $100 \%$ storage efficiency. For those runs where the storage efficiency was other than $100 \%$ the stored energy was set to $6 \mathrm{GWh}$ divided by the output efficiency to give a higher initial storage value. The ensured that the system could run for the first six hours of the model period before the sun rises on the first day. Values lower than 6 hours of storage prevented the model from converging as there was no way for the demand in the first six hours of the year (before sunrise) to be met. Higher values of initial storage had little effect on the model results.

It should be noted that the model implementation does not account for a real solar panel technology, but assumes we can convert the sun's energy to electricity. The model also does not consider the orientation of the solar panels, or any energy required to rotate the panels, but assumes that the technology exists to convert the solar energy into electricity. The model also does not account for the worst-case situation, but uses a typical meteorological year for the analysis. As such, this study is a good first approximation of the required storage capacity required for a system, and illustrates the scale of the problem, but does not provide a definitive answer to the question of how much storage is required in any given application.

\section{RESUlts}

As noted in the introduction, there are three research objectives that are addressed. First, can a 5 GWe solar $(20 \%$ capacity factor) installation meet a $1 \mathrm{GW}$ demand (and how much storage is required to do so)? Second, how does the storage efficiency impact the required storage capacity as well as the required solar panel installation size? And third, how do storage capacity limitations impact the required solar panel installed capacity?

\section{A. 1 GW Demand with 5 GW Solar Installation?}

With $100 \%$ efficient, free storage, with no capacity restrictions, it is possible to get down to almost $5 \mathrm{GW}$ of required solar installed capacity, but it is difficult. The solar output in Flagstaff is lowest during the winter and highest during the summer. To get down to a solar capacity of $5 \mathrm{GW}$ it is necessary to shift the year such that it runs from July to June rather than January to December. This allows the higher-output months of July and August to charge the storage system to meet the demand in the lower output winter months (December/January).

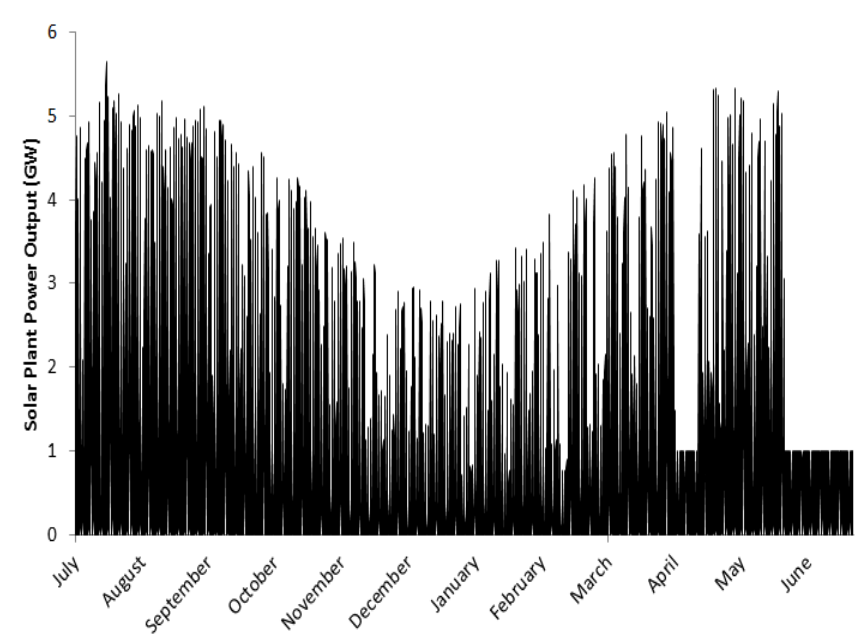

Fig. 4. Power output for solar for one year (base case).

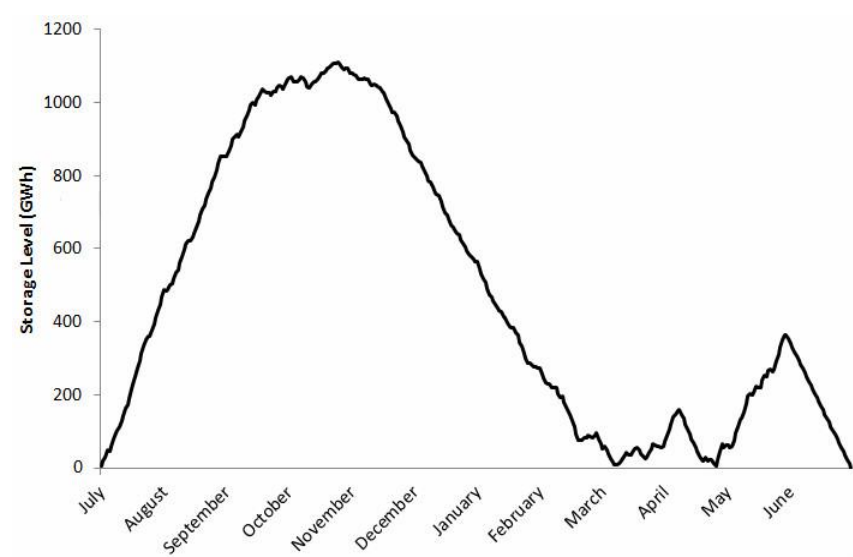

Fig. 5. Stored energy for solar for one year (base case).

Fig. 4 shows the power output over the course of a year (July to June) for the solar panel, and Fig. 5 shows the stored energy in GWh. From Fig. 4 it can be observed that the highest output from the solar installation is $5.63 \mathrm{GW}$ (mid-July), slightly higher than the $5 \mathrm{GW}$ system assumed by Myhrvold and Caldeira. It is clear that, with the right start date in the year, it would be possible to get down to a $5 \mathrm{GW}$ installed capacity with a $100 \%$ efficient storage system.

From the right hand side of Fig. 4 there are some time periods (and most of the month of June) where the output 
from the solar panels is no more than $1 \mathrm{GW}$. This is the result of the model having excess energy stored from previous time periods, and the model choosing to use the stored energy to meet the demand and running the storage down to zero at the end of the year (as can be seen in Fig. 5, which ends the year at zero stored energy) rather than taking more energy from the solar panels. In a real system, this excess energy would be kept (if possible) such that it could be used in future years. The excess energy is due to the system installing $5.63 \mathrm{GW}$ instead of $5 \mathrm{GW}$ (to meet the demand earlier in the year) and as such, it is used at the end of the year.

It can be seen from Fig. 5 that over $1.1 \mathrm{TWh}(1100 \mathrm{GWh})$ of storage is required to allow the $5.63 \mathrm{GW}$ solar installation to run the $1 \mathrm{GW}$ load in the model. As a comparison, the storage facility touted as "the largest battery in the world", the Bath County Pumped Storage Station in Virginia has a storage capacity of $30.9 \mathrm{GWh}(3,003 \mathrm{MW}$ X 10.3 hours of operation) [9], [10], a factor of 35 times smaller. Large scale storage such as that provided by the Bath County Pumped Hydro Storage Station is only feasible where there are opportune geological formations, and the costs are generally large, both in terms of monetary value and in terms of environmental impact. The Bath County station took eight years to construct at a final cost of $\$ 1.6$ Billion (1985 dollars) and flooded approximately 350 hectares of land [9].

The above results were based on $100 \%$ efficient storage. Realistic storage efficiencies will impact the results.

\section{B. Storage Efficiency and Solar Installation Requirements}

Fig. 6 shows the impact of efficiency on the required solar panel installation and the available storage capacity. As is to be expected, as the round trip efficiency of the storage system decreases, the required installed solar and the required storage capacity increase. The increase is small at high efficiencies, but at low efficiencies the increase becomes quite significant as the required capacities will tend to infinity at $0 \%$ efficient storage.

The round-trip efficiency of large scale storage facilities such as the Bath County Pumped Hydro facility currently range between $70 \%$ and $80 \%$ [11]. At $70 \%$ efficient storage, from Fig. 6, the required installed solar capacity is $6.97 \mathrm{GW}$ and the required storage capacity is $1.21 \mathrm{TWh}$. The solar panel installation size is still close to the $5 \mathrm{GW}$ assumed by Myrhvold and Caldeira, but the storage size is still significantly larger than what is reasonably available or buildable given geological constraints.

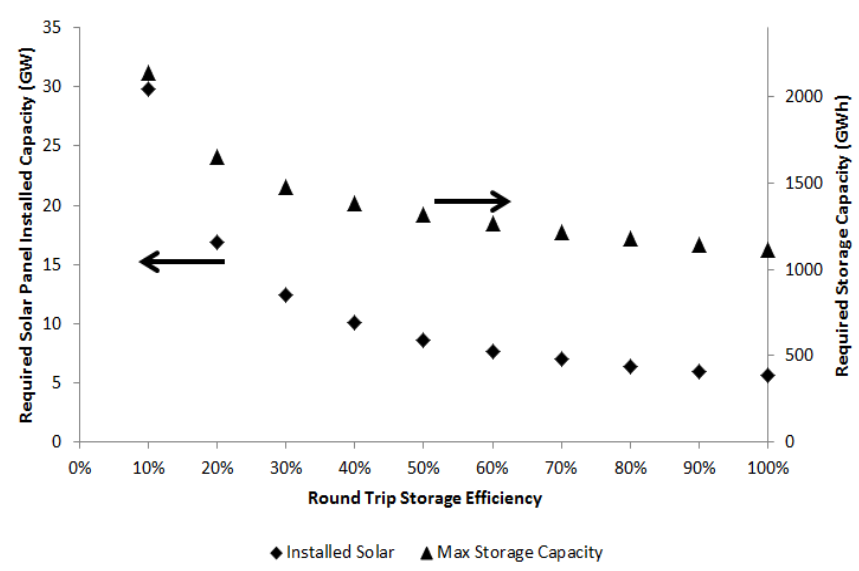

Fig. 6. Required solar installation and storage capacity vs. storage efficiency.

\section{Storage Limitations and Solar Installation Requirements (100\% Efficient)}

Given that the required storage capacity to allow $5 \mathrm{GW}$ of solar power to meet a steady $1 \mathrm{GW}$ of demand is $1.1 \mathrm{TWh}$ (at $100 \%$ efficient, higher at lower efficiencies), more than 35 times larger than the largest battery in the world, it makes sense to see how much installed solar capacity is needed with more realistic levels of storage capacity. The MaxStorageSize parameter discussed earlier was used to restrict the available storage capacity to values between 12 GWh (the lowest size that allowed the solar installation to still meet the demand) to $1.1 \mathrm{TWh}$ (unrestricted). This was first done for $100 \%$ efficient storage, as shown in Fig. 7. For $100 \%$ efficient storage, the $12 \mathrm{GWh}$ value is equivalent to saying that there is barely enough storage to make it through a single night, and that the storage system must be fully recharged each and every day, no matter how cloudy or un-ideal the weather is for solar generation. At this low value of storage the required installed solar capacity is very high, at $59.6 \mathrm{GW}$ such that the lowest solar insolation day can charge the storage fully, even when the insolation on that day is very low (on, for example, a really rainy, cloudy day in mid-winter).

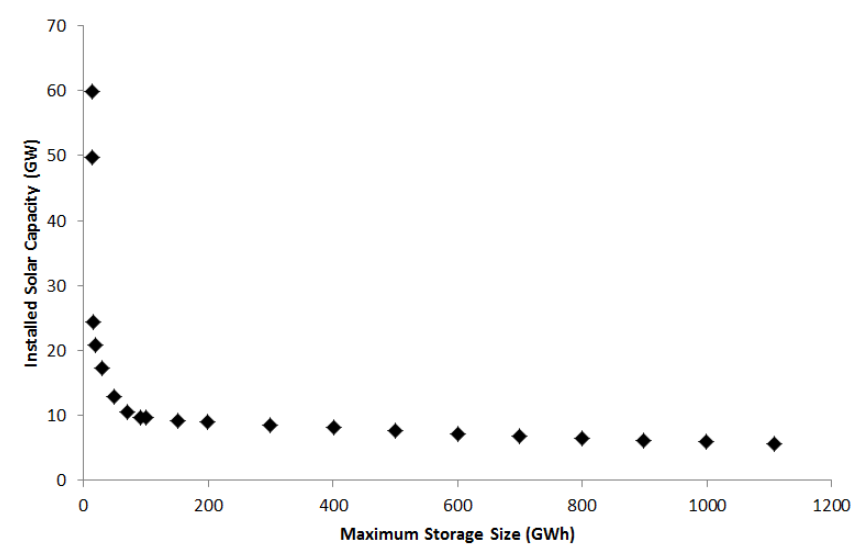

Fig. 7. Installed capacity required for restricted storage size (100\% efficient).

Fig. 7 shows that, for a $100 \%$ efficient storage system, the installed capacity of solar required to power a $1 \mathrm{GW}$ load between an available storage size of $1.1 \mathrm{TWh}$ (unlimited) and an available storage capacity of $0.1 \mathrm{TWh}$ (an order of magnitude smaller), the required solar capacity nearly doubles, from $5.63 \mathrm{GW}$ to $9.56 \mathrm{GW}$, respectively in a nearly linear fashion. When the available storage goes below 0.1 TWh, however, the required solar installed capacity starts increasing dramatically. This dramatic increase is due to the fact that, rather than providing long-term/seasonal storage the system now has to deal with short term weather events. With more than $0.1 \mathrm{TWh}$ of storage the system has the ability to average out these short term events and they therefore do not significantly impact the required capacity. 0.1 TWh corresponds to roughly 4 days' worth of storage for a $1 \mathrm{GW}$ load (100 GWh is $\sim 4 \times 24$ hours $\times 1 \mathrm{GW}$ ). This length of storage is likely the duration of a winter storm event with very little insolation during that time.

As discussed above, the Bath County Pumped Hydro station is the "largest battery in the world". It is illustrative to consider how much solar capacity is required if it was possible to re-purpose the entire Bath County facility to 
provide storage for a solar installation to meet at $1 \mathrm{GW}$ baseload. For this case, assuming that Bath County is $100 \%$ efficient, the installed solar capacity required is $17.2 \mathrm{GW}$, a factor of 3.5 larger than the assumption made by Myhrvold and Caldeira, assuming that it can operate at $100 \%$ efficiency. This is a significantly larger installation than that calculated by assuming a $20 \%$ capacity factor and means that both the cost and environmental impact of the installation is 3.5 times larger.

\section{Impact of Restricted Storage Size and Efficiency Together}

Since realistic storage systems have both size limitations and efficiency limitations, the model was run with restricted storage size for various levels of storage efficiency. The results for $100 \%$ down to $50 \%$ efficient storage, at levels between $12 \mathrm{GWh}$ and $50 \mathrm{GWh}$ are shown in Fig. 8. $12 \mathrm{GWh}$ is as low as it is possible to go (and then, only for the $100 \%$ efficient system), since we need to be able to run through the night for any feasible system. Any lower and the model do not converge due to an inability to run overnight. The plot was cut off at $50 \mathrm{GWh}$ so the detail of the dramatic increase in installed capacity required on the left hand side can be seen. This increase is due to the fact that, at low storage size, the system must be able to fully charge the storage system even during the worst insolation winters day.

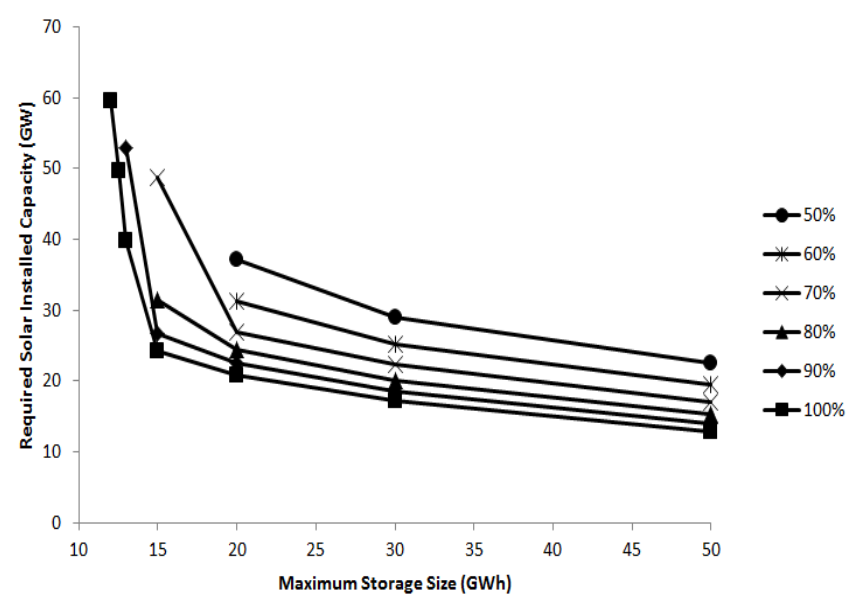

Fig. 8. Required solar installation with restricted storage size and varying efficiency.

For the $100 \%$ efficient storage system it was possible to get down to $12 \mathrm{GWh}$ of storage (equivalent to 12 hours of storage) and still run the system. 12 hours of storage seems to be the minimum level for the system as this is just enough to allow the system to run through a 12 hour winter night. At $90 \%$ efficient storage, it is no longer possible to run the system at $12 \mathrm{GWh}$ of storage, but it can run at $13 \mathrm{GWh}$ of storage. This makes sense as, with a lower efficiency, the $12 \mathrm{GWh}$ cannot provide enough storage to make it through the night while 13 GWh can just do so. At $80 \%$ and $70 \%$ efficiency $13 \mathrm{GWh}$ is not enough to make it through a winter night, and the lowest feasible capacity is $15 \mathrm{GWh}$, while for $60 \%$ and $50 \%$ storage, the lowest feasible capacity was $20 \mathrm{GWh}$.

Again, consider if a realistic Bath County Pumped Hydro Facility was re-purposed entirely to work with a solar installation to meet a $1 \mathrm{GW}$ demand. In this case, the capacity of $30 \mathrm{GWh}$ is available at between $70 \%$ and $80 \%$ efficiency. This requires between $20.2 \mathrm{GW}$ (80\% efficiency) and 22.3
GW (70\% efficiency) of installed capacity, 4 and 4.5 times larger than the size assumed by Myhrvold and Caldeira.

Assuming only half the Bath County facility is available to serve the solar panel installation (if, for example, we wanted to serve two equivalent solar installations with the Bath County facility), the required installed capacity goes up to 24.5 GW (80\% efficiency) and 27.0 GW (70\% efficiency), 5 and 5.5 times larger than assumed by Myhrvold and Caldiera.

\section{CONCLUSION}

The assumption that, as long as a system produces 8.76 TWh of electricity over the course of a year, it can replace a coal or fossil fuel powered generation station that produces the same amount of energy is flawed. With a realistic storage system size, and realistic storage system efficiency, to power a $1 \mathrm{GW}$ load, between 20 and $25 \mathrm{GW}$ of installed solar capacity are required plus between 15 and $30 \mathrm{GWh}$ of storage capacity. The capital infrastructure required to allow a solar installation to meet the power output of a $1 \mathrm{GW}$ coal plant is therefore around an order of magnitude larger than what is commonly assumed (5 times more installed solar capacity, plus around the same amount of infrastructure to build the storage facility).

The analysis in this paper was performed for a $1 \mathrm{GW}$ demand, and required nearly the entire capacity of the largest battery in the world. The assumption Myhrvold and Caldeira make that they can build out $5 \mathrm{TW}$ of solar capacity to replace the $1 \mathrm{TW}$ would require around 1.2 PWh (1200 TWh) of available storage (at $80 \%$ efficiency), which is unlikely to be feasible, even with advancing technologies. Storage is a huge problem for the large scale implementation of variable energy resources such as solar and wind.

It should be noted that this work was done based using solar insolation data for Flagstaff, Arizona. Arizona is generally a sunny state, but Flagstaff is located at 35 degrees $\mathrm{N}$ latitude. As such, the yearly variation is higher than for a similar installation nearer the equator (though it would be lower than a similar installation farther from the equator). The lower the yearly variation, the lower the overall storage requirements would be, though weekly weather related variations would still occur. As such, countries such as Canada would need larger storage systems while countries nearer the equator would need less.

This work assumed the same capacity factor (20\%) as Myhrvold and Caldeira. Actual capacity factors for existing solar power facilities in the United States range from $2.5 \%$ in the winter to over $30 \%$ in the summer, with an average of around $20 \%$. The California solar facility discussed by Wilson has an expected capacity factor of around $30 \%$, but it is not clear if this expectation will be met by the facility in operation (30\% is quite high compared to similar installations in other parts of the world). Obviously, a higher capacity factor would reduce the size of the solar installation and potentially the size of the storage system to support it.

Overall, this study is a good first approximation to the required storage capacity required for a system, and illustrates the scale of the problem, but does not provide a definitive answer to the question of how much storage is required in a given application. There are many factors that would need to be specified to get an estimate of the actual 
storage (and of the actual installed generation capacity) required for a real system, including the actual technologies for generation and storage, the nature of the variable resource (seasonal, weekly, daily variation), the type of resource (solar, wind, wave, etc.) and the amount of storage that can reasonably be implemented for the given location.

\section{FUTURE WORK}

A number of directions can be taken with this work. First, it would be good to look at the storage implications for wind, tidal and wave power to see if the storage requirements for these technologies would be similar to the storage requirements for solar. Second, the implementation of commercially available solar power and storage technologies would give results that would be easier for the general public to understand, allowing for a more general audience for the work. Finally, extending the storage requirements to include the analysis by Myhrvold and Caldeira looking at the climate change implications of these systems would be interesting.

\section{REFERENCES}

[1] N. P. Myhrvold and K. Caldeira, "Greenhouse gases, climate change and the transition from coal to low-carbon electricity," Environ. Res. Lett., vol. 7, no. 1, p. 014019, Mar. 2012.

[2] R. Socolow, " 40 yr. phase-out for conventional coal? If only!" Environ. Res. Lett., vol. 7, no. 1, p. 011009, Mar. 2012.

[3] R. Wilson, "How big is the world's biggest solar farm?" the Energy Collective, 09-Apr-2014.

[4] National Renewable Energy Lab. (2014). National solar radiation Data base. [Online]. Available: http://rredc.nrel.gov/solar/old_data/nsrdb/.
[5] M. Howells, H. Rogner, N. Strachan, C. Heaps, H. Huntington, S Kypreos, A. Hughes, S. Silveira, J. DeCarolis, M. Bazillian, and A. Roehrl, "OSeMOSYS: The open source energy modeling system," Energy Policy, vol. 39, no. 10, pp. 5850-5870, Oct. 2011.

[6] M. Welsch, M. Howells, M. Bazilian, J. F. DeCarolis, S. Hermann, and H. H. Rogner, "Modelling elements of smart grids - enhancing the OSeMOSYS (open source energy modelling system) code," Energy, vol. 46, no. 1, pp. 337-350, Oct. 2012.

[7] GNU MathProg. [Online]. Available: http://lpsolve.sourceforge.net/5.5/MathProg.htm

[8] Wikipedia. Bath county pumped storage station. (2014). [Online]. Available:

http://en.wikipedia.org/wiki/Bath_County_Pumped_Storage_Station.

[9] Wikipedia. List of energy storage projects. (2014). [Online]. Available: http://en.wikipedia.org/wiki/List_of_energy_storage_projects.

[10] Wikipedia. Pumped hydro storage. (2014). [Online]. Available: http://en.wikipedia.org/wiki/Pumped-storage_hydroelectricity.

[11] Electric Power Monthly with Data for July 2014, Energy Information Agency, Sep-2014.

Taco A. Niet completed his bachelor degree of engineering in 1998 and his masters of applied science (mechanical engineering) degree in 2002, both at the University of Victoria (UVic) in Victoria, British Columbia, Canada. He is currently pursuing his $\mathrm{Ph} . \mathrm{D}$. degree in mechanical engineering at the Institute for Integrated Energy Systems (IESVic) at the University of Victoria.

$\mathrm{He}$ is the program head of the bachelor of mechanical engineering at the British Columbia Institute of Technology (BCIT) in Burnaby, British Columbia, Canada. His research interests include renewable energy, sustainability, energy systems modelling and energy policy. He also acts as the mentor for the BCIT Underwater Remotely Operated Vehicle (ROV) Team and has successfully taken students to the MATE International ROV Competition six times.

Mr. Niet is a member of the Marine Technology Society and is registered as a professional engineering with the Association of Professional Engineers and Geoscientists of BC. 